\title{
Performance of Opportunistic Distributed Power Control with QoS Guarantee in Discrete Power Domain
}

\author{
F. de S. Chaves, R. B. Santos, F. R. P. Cavalcanti and R. A. de Oliveira Neto
}

\begin{abstract}
Resumo-O problema de controle de potência descentralizado em um sistema de comunicação sem-fio genérico pode ser visto como uma competição entre os nós de comunicação. Neste artigo, nós formulamos uma estrutura para o problema com a aplicação de teoria dos jogos não-cooperativos e de conceitos de alocação de recursos oportunista, em que a satisfação de requisitos de Qualidade de Serviço (QoS) é considerada. Como habitual, assume-se um domínio contínuo para a potência de transmissão no desenvolvimento do algoritmo de controle de potência. Entretanto, o desempenho do algoritmo é avaliado nos domínios contínuo e discreto da potência de transmissão por meio de simulações computacionais.
\end{abstract}

Palavras-Chave-Controle de potência distribuído, controle de potência oportunista, satisfação de restrições de QoS, teoria dos jogos, domínio discreto de potências.

Abstract-The decentralized power control problem in a generic wireless communication system can be seen as a competition among the communication nodes. In this paper, we formulate a framework to the problem with the application of noncooperative game theory and opportunistic resource allocation concepts, where the satisfaction of Quality of Service $(Q \circ S)$ requirements is considered. As usual, continuous transmit power domain is assumed for derivation of the power control algorithm. However, the algorithm's performance is evaluated in continuous and discrete transmit power domains through computer simulations.

Keywords-Distributed power control, opportunistic power control, QoS satisfaction, game theory, discrete power domain.

\section{INTRODUCTION}

Wireless communication systems may be generically modeled as a set of communication nodes with a limited amount of radio resources. A typical system requires an efficient interference management, since high interference levels deteriorate the quality of the links and, consequently, affect the system capacity [1]. Furthermore, some communication nodes may be supplied by batteries, whose lifetime is another important aspect. Transmitter power control is an essential tool for coping with the problem of interference management.

Distributed or decentralized power control is of special interest and importance, since it allows the use of only local

F. de S. Chaves is with Department of Communications, School of Electrical and Computer Engineering, University of Campinas - UNICAMP, Campinas, Brazil, E-mail: fabiano@decom.fee.unicamp.br. He was scholarship supported by FAPESP.

R. B. Santos, F. R. P. Cavalcanti and R. A. de Oliveira Neto are with Department of Teleinformatics Engineering, Federal University of Ceará UFC, Fortaleza, Brazil, E-mails: \{brauner, rodrigo, neto\}@gtel.ufc.br. R. B. Santos and R. A. de Oliveira Neto were scholarship supported by FUNCAP. F. R. P. Cavalcanti was partly funded by CNPq, grant no. 304477/2002-8. information for determining a suitable transmit power [2]. The distributed control corresponds to a self-optimization process, since the transmit powers of all nodes can not be jointly determined by a central entity. This motivates the application of noncooperative game theory [3], which begun to be explored in this context recently, as discussed in [4].

Conventional power control has as objective to provide a stable link condition to each transmitter/receiver pair by increasing the transmit power when the link condition is bad. For this purpose, many power control algorithms have been developed, as the classical Distributed Power Control (DPC) algorithm [2], which tracks a fixed target QoS. This approach is adequate for services with hard QoS requirements, like realtime services. On the other hand, the number of services which can tolerate larger delay has increased. The relaxed QoS restrictions of elastic (or best effort) services allow the application of opportunistic resource allocation.

Conventional and opportunistic power control have opposite philosophies. An opportunistic power control is essentially characterized by the idea of exploiting the quality of communication links [5], [6], [7]. In favored links (large channel gain and low interference), the transmit power must be increased in order to transmit more information. In [5], Xiao et al. propose a Utility-Based Power Control (UBPC) with the same structure of DPC algorithm, but with an adaptive target QoS (a decreasing function of the effective interference).

A different opportunistic framework is proposed by Sung and Leung et al. [6], [7], where the figure of a target QoS is absent. In this case, the transmit power update is inversely proportional to the effective interference. The algorithm proposed in [7] is a fair version of that proposed in [6].

In this paper, we present a different framework for the distributed power control problem, where an opportunistic power allocation is simultaneously considered with the satisfaction of flexible Quality of Service (QoS) requirements [8]. The proposed power control algorithm is derived from a multistage nonzero-sum noncooperative game, developed, as usual, in the context of continuous transmit power domain. In order to verify the feasibility of its employment in practical systems, where power control works over discrete domain, we evaluate its performance in terms of convergence, QoS satisfaction and energy efficiency in both continuous and discrete transmit power domains.

The paper is organized as follows. The system model is presented in Section II. In Section III, the opportunistic scheme with QoS guarantee is explained. Derivation of the power 
control algorithm is presented in Section IV. Section V presents the implemented discrete versions of the power control algorithm and illustrates their performance through simulation results. The conclusions of the paper are given in Section VI.

\section{SySTEM MODEL}

In this work, a generic wireless communication system is modeled as a set of $N$ co-channel radio links (transmitter/receiver pairs), $\mathbf{N}=\{1,2, \ldots, N\}$. We use $k$ as the discrete time index. For all $i, j \in \mathbf{N}, p_{j}(k)$ denotes the power used by transmitter of link $j ; G_{i j}(k)$ is the path gain between transmitter $j$ and receiver $i ; I_{j}(k)$ represents the interference-plusnoise power which affects the communication of radio link $j$. Then, the Signal-to-Interference-plus-Noise Ratio (SINR) perceived in the receiver of link $j, \rho_{j}(k)$, is given by:

$$
\rho_{j}(k)=\frac{G_{j j}(k)}{I_{j}(k)} p_{j}(k),
$$

where $I_{j}(k)$ is defined as:

$$
I_{j}(k)=\sum_{l=1}^{N}\left[p_{l}(k) G_{j l}(k)\right]+\sigma_{j}^{2}, l \neq j,
$$

with $\sigma_{j}^{2}$ as the average AWGN power in receiver $j$. In an abuse of notation, we will use $G_{j}$ in the place of $G_{j j}$ to represent path gain in link $j$. Then, SINR can be written as:

$$
\rho_{j}(k)=\frac{G_{j}(k)}{I_{j}(k)} p_{j}(k) .
$$

Quality of Service (QoS) is a quality measure commonly related to throughput, delay or Bit Error Rate (BER). QoS requirements are usually mapped into SINR requirements. In this work, we are concerned with services with throughput restrictions. In this case, assuming the use of adaptive coding, for any link $j$ the relation between instantaneous throughput and SINR is established by the ${ }^{1}$ Shannon's capacity formula:

$$
R_{j}=W \log _{2}\left(1+\rho_{j}\right),
$$

where $\mathrm{W}$ is the channel bandwidth.

\section{Opportunistic POWER CONTROL Scheme With QOS GUARANTEE}

The proposed opportunistic power control scheme is based on an adaptive target SINR, as in [5]. However, we use as link quality indicator the transmit power level instead of the effective interference. This is a good indicator, since for achieving a given SINR, transmitters in favored links use low power.

An opportunistic approach must determine higher target SINRs for favored transmitters and lower SINRs for transmitters in bad situations. In addition to the opportunism, we are concerned about the satisfaction of throughput (SINR)

\footnotetext{
${ }^{1}$ The use of the Shannon's formula may be optimistic, but it is in accordance with recent breakthrough research in coding theory such as turbo codes. Moreover, a slight change in this mapping relationship does not change the conclusions of our work.
}

restrictions, i.e., in the proposed scheme a desired throughput (SINR) range is established. Any non-increasing target SINR function of transmit power may implement the opportunistic behavior discussed above. We adopt a sigmoid function due to its softness and mathematical tractability. Such function is defined below for each link $j$ :

$$
\rho_{j}^{t}(k+1)=\frac{\alpha}{1+\beta p_{j}^{2}(k+1)},
$$

where $\alpha$ and $\beta$ are constant non-negative parameters, and $\rho_{j}^{t}$ and $p_{j}$ are the SINR to be targeted and the transmit power, respectively. Fig. 1 illustrates the target SINR adaptive rule.

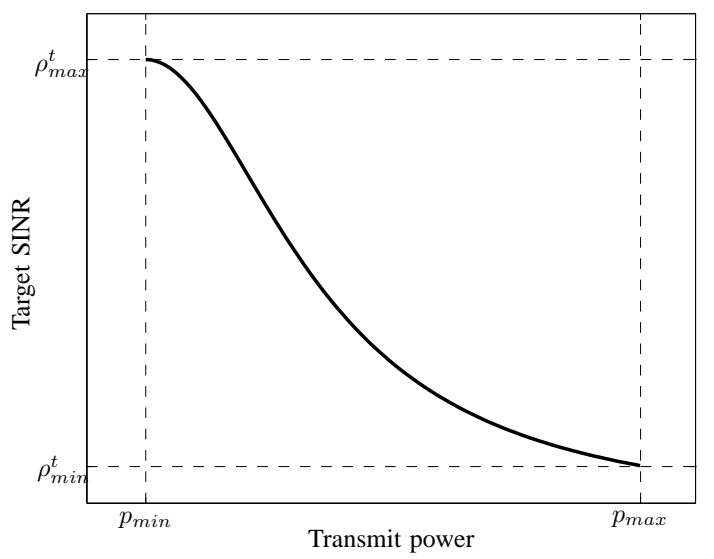

Fig. 1. Target SINR as a function of transmit power, with power and QoS restrictions (maximum and minimum transmit powers, $p_{\max }$ and $p_{\min }$, and maximum and minimum target SINR values, $\rho_{\text {max }}^{t}$ and $\rho_{\text {min }}^{t}$ ).

Parameters $\alpha$ and $\beta$ are defined in terms of transmit power restrictions and QoS (SINR) requirements:

$$
\begin{array}{r}
\alpha=\rho_{\text {max }}^{t}\left(1+\beta p_{\text {min }}^{2}\right), \\
\beta=\frac{\rho_{\text {min }}^{t}-\rho_{\text {max }}^{t}}{\rho_{\text {max }}^{t} p_{\text {min }}^{2}-\rho_{\text {min }}^{t} p_{\text {max }}^{2}} .
\end{array}
$$

\section{Power Control Game}

We formulate the power control problem with power and QoS restrictions as a multi-stage nonzero-sum noncooperative game [3]. The transmitters are the players, whose decision variables are their transmit powers. The $k$ th stage corresponds to the $k$ th power control actuation. Moreover, the objective of each player is to minimize an average long horizon cost, with costs at each stage contributing to this average cost.

Although the natural dynamic aspect of the problem, we use the framework of static games to solve it, where in each stage the players minimize their cost functions only under past information. This is a rather "myopic" behavior, but it makes sense, since the players have no idea as to how many stages the game comprises. Then, minimizing their current cost functions is a risk-aversing behavior. In order to propose a target tracking power control with an adaptive target SINR, we use a suitable cost function $c_{j}$ [9]:

$$
\min _{p_{j}(k+1) \in P_{j}}\left\{c_{j}\left(p_{j}(k+1), \mathbf{p}_{-j}(k+1)\right)=\left[\rho_{j}^{t}-\rho_{j}\right]^{2}\right\},
$$


where for each player $j, P_{j}=\left[p_{\min }, p_{\max }\right]$ is the continuous set of power values and $\mathbf{p}_{-j}(k+1)$ denotes the power vector of players other than the $j$ th one in time $(k+1)$.

The target SINR $\rho_{j}^{t}$ is determined according to the opportunistic scheme given by (5). Then, at time $k$, each player must jointly determine its target SINR and its transmit power for the next time instant, since $\rho_{j}^{t}(k+1)$ is a function of $p_{j}(k+1)$.

\section{A. Nash equilibrium point}

In decentralized power control, players define their transmit powers individually. However, the transmit power that optimizes the individual cost depends on the transmit powers of all other players. Therefore, it is desirable a set of powers where each player is satisfied with its cost. We use Nash equilibrium concepts, which provide predictable and stable outcomes through self-optimization. The Nash equilibrium (NE) solution is a point where no player wishes to deviate from. Formally, a power vector $\mathbf{p}^{*}(k)=\left[p_{1}^{*}(k), \ldots, p_{N}^{*}(k)\right]$ is a NE point of our power control game if, for each $j \in \mathbf{N}$ :

$$
c_{j}\left(p_{j}^{*}(k), \mathbf{p}_{-j}^{*}(k)\right) \leq c_{j}\left(p_{j}(k), \mathbf{p}_{-j}^{*}(k)\right) .
$$

The conditions for the existence of a NE solution are established in the following theorem [3] (pp. 173-174):

Theorem 1: For each $j \in \mathbf{N}$, let $P_{j}$ be a closed, bounded and convex subset of a finite-dimensional euclidian space, and the cost functional $c_{j}: P_{1} \times \cdots \times P_{N} \longrightarrow \mathbb{R}$ be jointly continuous in all its arguments and strictly convex in $p_{j}$ for every $p_{l} \in P_{l}, l \neq j$. Then, the associated nonzero-sum game admits a Nash equilibrium solution.

Using (3) and (7), we express the cost function $c_{j}$ in terms of the transmit power of all players in (9). There and in the following developments, the time instant indicator $(k+1)$ will be omitted. It can be observed that $c_{j}$ is a continuous function, since $I_{j}$ is positive for each $j \in \mathbf{N}$.

$$
c_{j}=\left(\frac{G_{j}}{I_{j}} p_{j}\right)^{2}-2 \alpha \frac{G_{j} p_{j}}{I_{j}\left(1+\beta p_{j}^{2}\right)}+\left(\frac{\alpha}{1+\beta p_{j}^{2}}\right)^{2} .
$$

The strategy space $P_{j}=\left[p_{\min }, p_{\max }\right]$ is a closed, bounded and convex subset of the euclidian space $\mathbb{R}$, for all $j \in \mathbf{N}$. Considering the necessary optimality condition for $c_{j}$, its first order derivative with respect to transmit power is zero. Since target SINR is a non-decreasing function of transmit power and the SINR is an increasing function of transmit power, the necessary optimality condition becomes:

$$
\rho_{j}^{t}-\rho_{j}=0
$$

After some algebraic manipulation, we can rewrite (10) as:

$$
p_{j}^{3}+\frac{1}{\beta} p_{j}-\frac{\alpha I_{j}}{\beta G_{j}}=0 .
$$

In order to solve the third order polinomial equation we use the Tartaglia's method [10], which provides a real solution. Applying Tartaglia's method to (11), we obtain the strategy by which each player achieves the NE point. This strategy is given by (with time instant indicators):

$$
\begin{aligned}
p_{j}(k+1) & =\sqrt[3]{\frac{\alpha I_{j}(k+1)}{2 \beta G_{j}(k+1)}+\sqrt{\left(\frac{\alpha I_{j}(k+1)}{2 \beta G_{j}(k+1)}\right)^{2}+\frac{1}{(3 \beta)^{3}}}} \\
& +\sqrt[3]{\frac{\alpha I_{j}(k+1)}{2 \beta G_{j}(k+1)}-\sqrt{\left(\frac{\alpha I_{j}(k+1)}{2 \beta G_{j}(k+1)}\right)^{2}+\frac{1}{(3 \beta)^{3}}}} .
\end{aligned}
$$

The second-order derivative of $c_{j}$ may be shown to be strictly positive in the NE point, characterizing it as a local minimum. However, in the large set of situations we have observed, $c_{j}$ is strictly convex, configuring the NE point as the global minimum of $c_{j}$.

\section{B. Convergence analysis of the NE solution}

The NE strategy given in (12) is unfeasible, since in time $k$ the necessary information for determining $p_{j}(k+1)$ is not available. However, a stability analysis of the NE point reveals that it is stable with respect to a special case of strategy deviation: the situation where players adjust their actions simultaneously in response to the more recent information. This situation is expressed by:

$$
\begin{aligned}
p_{j}(k+1) & =\sqrt[3]{\frac{\alpha I_{j}(k)}{2 \beta G_{j}(k)}+\sqrt{\left(\frac{\alpha I_{j}(k)}{2 \beta G_{j}(k)}\right)^{2}+\frac{1}{(3 \beta)^{3}}}} \\
& +\sqrt[3]{\frac{\alpha I_{j}(k)}{2 \beta G_{j}(k)}-\sqrt{\left(\frac{\alpha I_{j}(k)}{2 \beta G_{j}(k)}\right)^{2}+\frac{1}{(3 \beta)^{3}}}} .
\end{aligned}
$$

From the NE strategy given in (12), we can state that:

$$
p_{j}(k)=\arg \min _{p_{j}(k) \in P_{j}} c_{j}\left(p_{j}(k), p_{-j}(k)\right),
$$

and the power in the NE point, $p_{j}^{*}$, can be written as:

$$
\lim _{k \rightarrow \infty} p_{j}(k)=p_{j}^{*}, \quad \forall j \in \mathbf{N} .
$$

The commented special case of deviation (13) corresponds to:

$$
p_{j}(k+1)=\arg \min _{p_{j}(k+1) \in P_{j}} c_{j}\left(p_{j}(k+1), p_{-j}(k)\right) .
$$

It can be seen that the deviation (13) from the NE strategy, where the players adjust their transmit power in response to the status given in the previous power control actuation, iteratively converges to the NE point:

$$
\lim _{k \rightarrow \infty} p_{j}(k+1)=\lim _{k \rightarrow \infty} p_{j}(k)=p_{j}^{*}, \quad \forall j \in \mathbf{N} .
$$

Therefore, after a simple algebraic manipulation over (13), we can finally present the Opportunistic QoS-Guaranteed Distributed Power Control (OQ-DPC) algorithm:

$$
\begin{aligned}
p_{j}(k+1) & =\sqrt[3]{\frac{\alpha p_{j}(k)}{2 \beta \rho_{j}(k)}+\sqrt{\left(\frac{\alpha p_{j}(k)}{2 \beta \rho_{j}(k)}\right)^{2}+\frac{1}{(3 \beta)^{3}}}} \\
& +\sqrt[3]{\frac{\alpha p_{j}(k)}{2 \beta \rho_{j}(k)}-\sqrt{\left(\frac{\alpha p_{j}(k)}{2 \beta \rho_{j}(k)}\right)^{2}+\frac{1}{(3 \beta)^{3}}}}
\end{aligned}
$$

with $\alpha$ and $\beta$ parameters defined in (6), and with $\rho_{j}(k)$ representing the SINR of player $j$ in time $k$. 


\section{Simulation Results}

In order to illustrate the performance of the proposed OQ-DPC algorithm, we execute some computer simulations. According to previous discussions, the OQ-DPC algorithm is devoted to the opportunistic power allocation for services with flexible QoS requirements. Thus, we consider a service with throughput requirements in the range: 64 to $256 \mathrm{kbps}$.

The presented algorithm is, as usual, developed over a continuous transmit power domain. In practice, however, power controlled systems work with discrete power sets. Therefore, towards practical implementation, we are interested in the algorithm's performance in the discrete transmit power domain, taking as reference its performance in continuous time domain. Then, three different versions of the algorithm are considered:

- OQ-DPC: Presented algorithm. It is executed over a continuous transmit power domain.

- OQ-DPC (Discrete): OQ-DPC algorithm executed over a discrete transmit power set with resolution of $1 \mathrm{~dB}$. At each power control actuation, the transmit power given by the OQ-DPC is approximated to the nearer value in the discrete power set.

- OQ-DPC (Up/Down): OQ-DPC algorithm executed with restrictions on the step of the transmit power adaptation. At each power control actuation, the transmit power is increased or reduced in $1 \mathrm{~dB}$.

We perform computer simulations of a generic single-cell wireless system with a single central node placed in the cell center. A snapshot simulation model is adopted, where mobile terminals (users) are uniformly distributed over the cell area. In each snapshot, up to 600 power control iterations are performed, in intervals of $0.666 \mathrm{~ms}$.

Other simulation parameters are set as follows. We consider a cell radius of $1 \mathrm{~km}$ and omnidirectional antennas. A generic macro-cell path loss $(P L)$ model for suburban areas is assumed, where $P L(d)=129.4+35.2 \log _{10}(d)$, with distance $d$ between transmitter and receiver nodes expressed in kilometers. Shadowing is modeled as a zero-mean log-normal random variable with standard deviation of $7 \mathrm{~dB}$. Fast fading follows the Jake's model [11] with carrier frequency assumed $2 \mathrm{GHz}$. In all simulations, the noise power is set to $-103 \mathrm{dBm}$ and the maximum transmit power is limited to $21 \mathrm{dBm}$ with a dynamic power range of $70 \mathrm{~dB}$.

We initially analyze the aspect of convergence. Fig. 2 illustrates a sample of the SINR evolution of a mobile terminal in a system with 10 mobile terminals. Minimum and maximum transmit powers are used as initial power values and, in all the cases, the convergence values for the three versions of OQDPC algorithm are not affected by the initial power value. Similar curves were obtained for all other terminals.

The behavior observed in Fig. 2 was expected for OQ-DPC algorithm in continuous power domain, since the algorithm was analytically shown to converge to the NE solution of the presented power control game. However, simulation results show that the algorithm's versions in discrete power domain also converge essentially to the NE solution, although their intrinsic inaccuracy.

Other important aspect is the speed of convergence. It is

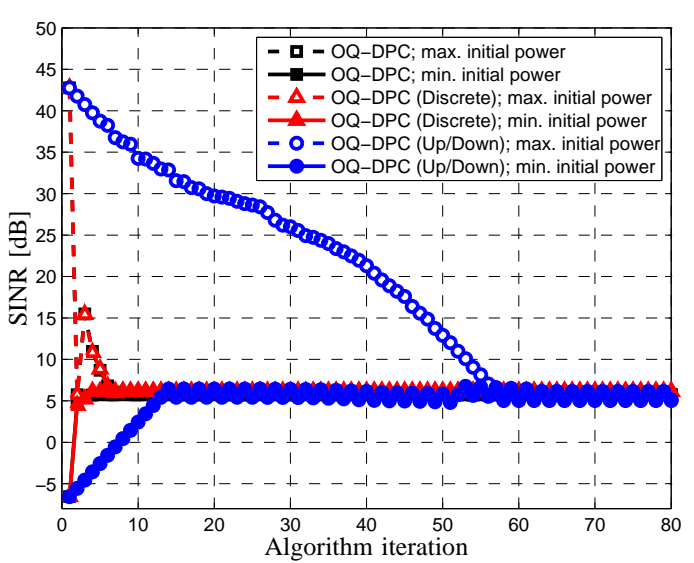

Fig. 2. Convergence curves of SINR: extreme initial power values.

clear that the limitation of OQ-DPC (Up/Down) in terms of its power adaptation step makes the convergence much slower than that of the other algorithm's versions. OQ-DPC and OQDPC (Discrete) have identical convergence speed.

Consequences of lower convergence speed of OQ-DPC (Up/Down) will be revealed in the following, where in order to obtain performance results statistically more representative, simulations of 10,000 snapshots are performed. For a channel coherence time much higher than the snapshot duration, the performance of the three algorithm's versions was observed to be basically the same (discarding the transient phase). They provide high throughput in the specified range (between 64 and $256 \mathrm{kbps}$ ). This is a very important conclusion, since it indicates a scenario where OQ-DPC algorithm can be wellapplied in practice without performance loss.

However, we also considered scenarios where terminals with vehicular mobility pattern $(10 \mathrm{~km} / \mathrm{h}$ or $50 \mathrm{~km} / \mathrm{h}$, i.e., Doppler spreads of $18.5 \mathrm{~Hz}$ and $92.5 \mathrm{~Hz}$, respectively) constitute the set of users. In such scenarios we can observe some performance degradation for OQ-DPC (Up/Down) in comparison with the performance of the other algorithm's versions.

As discussed, the proposed OQ-DPC algorithm has the task of to provide throughput in the specified range. Fig. 3 shows the Cumulative Distribution Function (CDF) curve of throughput obtained with the simulation of 25 mobile terminals in the system. In general, we observe that the throughput levels are mostly inside the specified range. Curves of OQ-DPC and OQDPC (Discrete) are similar for both terminal speeds.

One can see in Fig. 3(a) that for terminals with low speed the performance of OQ-DPC (Up/Down) algorithm is only slightly worse than that of the other ones. In the other hand, Fig. 3(b) shows a significant performance loss of OQDPC (Up/Down) for high $(50 \mathrm{~km} / \mathrm{h})$ speed, explained by its low capability of tracking channel variation. About $25 \%$ of instantaneous throughput is below $64 \mathrm{kbps}$.

Cumulative distribution of throughput contains important information, but further performance indicators may be observed. The energy efficiency is defined as the quantity of bits transmitted with a unit of energy, or the ratio between the average throughput (kbps) and the average transmit power $(\mathrm{mW})$. In Fig. 4, the average energy efficiency per terminal is 


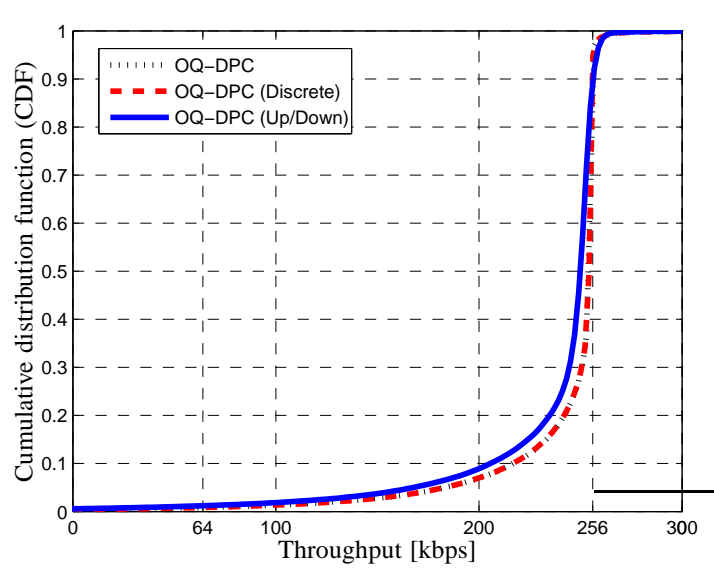

(a) Speed of mobile terminals: $10 \mathrm{~km} / \mathrm{h}$

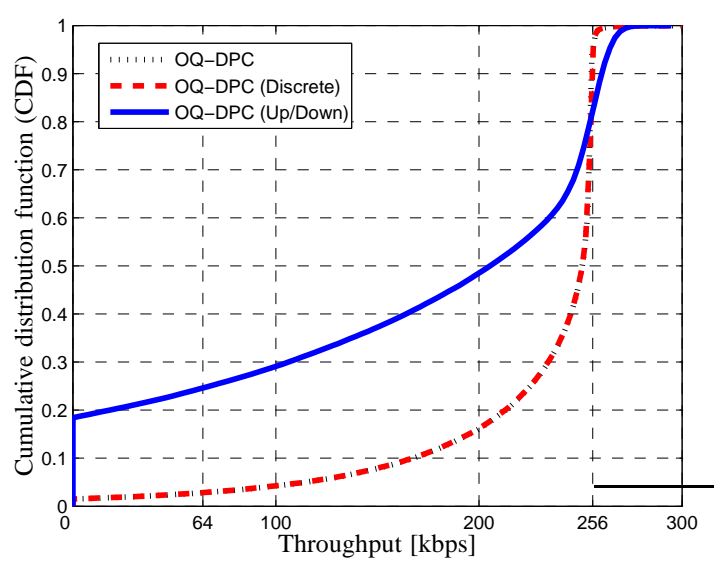

(b) Speed of mobile terminals: $50 \mathrm{~km} / \mathrm{h}$

Fig. 3. CDF curve of achieved throughput.

presented for different system loads.

Also in this case, OQ-DPC and OQ-DPC (Discrete) perform identically for both terminal speeds. A slight loss of energy efficiency is noted for OQ-DPC (Up/Down) in Fig. 4(a), for terminals with low speed. We have observed that in this case the achieved throughput per terminal is essentially the same for all algorithm's versions.

Fig. 4(b) shows the performance degradation suffered by OQ-DPC (Up/Down) in terms of energy efficiency for scenarios with high speed terminals. The loss is dramatic specially for high loaded systems. It was observed the use of more power resources with the attainment of lower throughput.

Finally, we verify the capability of the algorithm to maintain the achieved throughput above the threshold value. In practical systems, the minimum SINR corresponds the SINR required to maintain the minimum QoS. A margin $(1 \mathrm{~dB})$ below the minimum SINR is considered, in which QoS (throughput) is still assumed acceptable. Then, the average fraction of time in which the achieved throughput is below the minimum desired, $64 \mathrm{kbps}$, is calculated for all algorithms and shown in Fig. 5.

This figure brings results for both terminal speeds. As expected, high system loads and terminals with high speed produce more difficulties for the satisfaction of QoS requirements

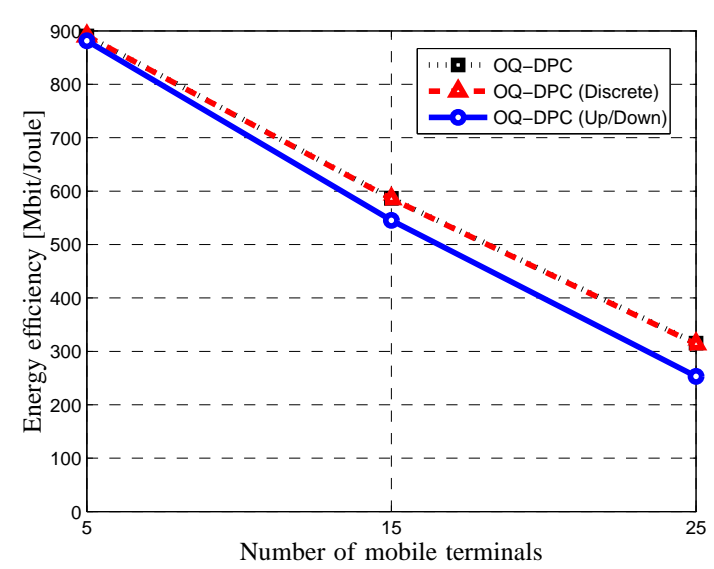

(a) Speed of mobile terminals: $10 \mathrm{~km} / \mathrm{h}$

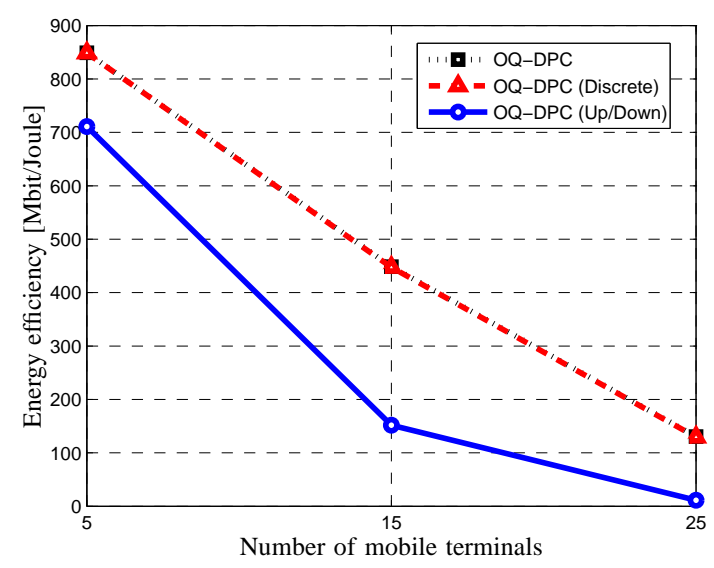

(b) Speed of mobile terminals: $50 \mathrm{~km} / \mathrm{h}$

Fig. 4. Average energy efficiency per terminal.

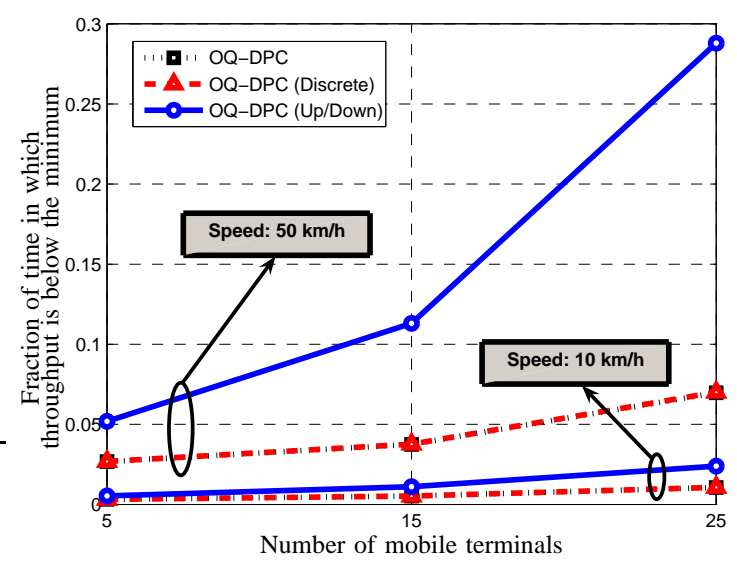

Fig. 5. Fraction of time in which throughput is below the minimum (64 kbps).

as a minimum throughput. This is observed for all considered algorithm's versions. Once more, OQ-DPC and OQ-DPC (Discrete) have the same performance. In the scenario of low terminal speed, the performance loss of OQ-DPC (Up/Down) algorithm compared to the other ones is small. However, as observed for previous performance indicators, OQ-DPC (Up/Down) suffers an important performance degradation due 
to its convergence behavior, which is critic when channel coherence time is small compared to snapshot duration.

\section{CONCLUSIONS}

In this paper, with the use of a game-theoretical framework, we propose an opportunistic distributed power control algorithm with power and QoS restrictions, the OQ-DPC algorithm. The algorithm is analytically shown to converge to the Nash equilibrium solution of the presented multi-stage noncooperative power control game. The extensive computer simulations indicate that it performs well for elastic services, providing high throughput levels inside the specified range, which constitutes the flexible QoS restrictions.

The algorithm presented in this work is, as usual, developed over a continuous transmit power domain. In practice, however, power controlled systems work with discrete power sets. Then, we evaluate the OQ-DPC algorithm performance in discrete transmit power domains through computer simulations in the context of a generic single-cell wireless system with a single central communication node, taking as reference the algorithm's performance in continuous time domain.

Results of simulations where channel coherence time is much higher than the snapshot duration show that OQ-DPC algorithm can be employed in discrete transmit power environments without performance loss. In scenarios with varying channels where mobile terminals move at $10 \mathrm{~km} / \mathrm{h}$, one can observe a slight worse performance of OQ-DPC (Up/Down) if compared to that of both other algorithm's implementations. Finally, the performance of OQ-DPC (Up/Down) suffers a dramatic loss in the context of high speed $(50 \mathrm{~km} / \mathrm{h})$ terminals.

\section{REFERENCES}

[1] J. Zander, "Distributed cochannel interference control in cellular radio systems," IEEE Trans. Veh. Technol., vol. 41, no. 3, pp. 305-311, Aug. 1992.

[2] G. J. Foschini and Z. Miljanic, "A simple distributed autonomous power control algorithm and its convergence," IEEE Trans. Veh. Technol., vol. 42, no. 4, pp. 641-646, Nov. 1993.

[3] T. Basar and G. J. Olsder, Dynamic Noncooperative Game Theory, SIAM, 2nd edition, 1998.

[4] A. B. MacKenzie and S. B. Wicker, "Game theory and the design of self-configuring, adaptive wireless networks," IEEE Commun. Magazine, vol. 39, no. 11, pp. 126-131, Nov. 2001.

[5] M. Xiao, N. B. Shroff, and E. K. P. Chong, "A utility-based powercontrol scheme in wireless cellular systems," IEEE/ACM Trans. Networking, vol. 11, no. 2, pp. 210-221, Apr. 2003.

[6] C. W. Sung and K. K. Leung, "A generalized framework for distributed power control in wireless networks," IEEE Trans. Information Theory, vol. 51, no. 7, pp. 2625-2635, Jul. 2005.

[7] K. K. Leung, C. W. Sung, and V. K. Bhargava, "An opportunistic power control algorithm with fairness," Proc. International Symposium on Information Theory, pp. 690-694, Sep. 2005.

[8] F. de S. Chaves, F. R. P. Cavalcanti, R. B. Santos, and R. A. de Oliveira Neto, "Opportunistic distribuited power control with QoS guarantee in wireless communication systems," Proc. IEEE Workshop on Signal Processing Advances in Wireless Communications (SPAWC), Jun. 2007

[9] R. A. de Oliveira Neto, F. de S. Chaves, F. R. P. Cavalcanti, and R. B Santos, Telecommunications: Advances and Trends in Transmission, Networking and Applications, chapter Power Control for Wireless Networks, pp. 83-108, University of Fortaleza Press, 2006.

[10] W. H. Beyer, CRC Standard Mathematical Tables and Formulae, CRC Press, 29th edition, 1991.

[11] W. C. Jakes, Microwave Mobile Communications, Wiley, 2nd edition, 1974. 